Abstract

\title{
Entelechy, Gyroscopes, and QBism: Developing a Quantum Metaphor for the Vital Force
}

\section{Lionel R Milgrom}

Program for Advanced Homeopathic Research, London, NW2 3ES, United Kingdom

milgromlr27412@gmail.com

Background Entelechy (e.g., Hahnemann's Vital Force, Vf), bears striking similarities to certain properties of quantum systems. Thus, the Vf is only indirectly observable via (centrifugally) expressed symptoms: a quantum property is only indirectly observable via its experimental effects. Consequently, a quantised Vf 'gyroscopic' metaphor is proposed where 'axial' rotation (represented by a wavefunction) undergoes dis-ease-induced retardation leading to Vf 'precession', i.e., symptom expression. Remedy-induced acceleration of axial rotation results in cure, via centrifugal removal of dis-ease, so precession/symptom expression ceases. The Vf 'gyroscope' is considered quantised partly because patients are observed during 'discreet' appointments, not continuously. Objective To develop this metaphor further by generating wavefunctions to represent the Vf in various states of dis-ease and health. Method Three wavefunctions are generated from secondary symptoms exhibited by the patient's Vf, as observed by the practitioner. Three more wave functions are generated from analogous (unobserved) hypothetical states, representing the Vf evolving from health into a precursor dis-eased state. Results and discussion The evolution of therapeutic/disease processes are imagined as transformations between these Vf wavefunctions, visualised as patient states on six of the nine points of an enneagram. The remaining three points represent practitioner states, 'entangled' with these processes. The Vf states may be divided into two groups one indirectly observable via expressed symptoms, the other hypothetical - separated by a notional therapeutic 'event horizon'. The practitioner, Janus-like, manages the therapeutic process by 'negotiating' between these two groups. Conclusion An interpretation of quantum theory called QBism (i.e., Quantum Bayesianism, in which a wavefunction represents only the total subjective information available to an assigning agent, not a shared separate objective reality), suggests these various Vf wavefunctions could represent Chalmers-like non-reductive information states, proposed as starting points for considering the influence of consciousness on the therapeutic process.

Keywords: Vital Force; quantised gyroscopic model; enneagram; QBism.

(C) International Journal of High Dilution Research.

Not for commercial purposes. 